\title{
Zerschlagene Siegel im mittelalterlichen Totenkult - vom „Altmetall“ zum Symbolakt
}

Zum Prozess der „Zerstörung von Geschriebenem“ kann die Archäologie des Mittelalters nur in seltenen Einzelfällen sachdienliche Hinweise liefern. Zunächst deshalb, weil Schrift generell nur selten in den Boden gelangt ist oder dort erhalten blieb und überlieferte Schriftzeugnisse des Mittelalters daher vorrangig von den Disziplinen der mittelalterlichen Geschichte und Kunstgeschichte erfasst und bearbeitet werden. Da die Archäologie Prozesse der Vergangenheit auf Basis ihrer materiellen Überreste untersucht, entzieht sich gerade die Zerstörung (von Schriftträgern) meist der direkten Erforschung. Der „stumme“ Charakter archäologischer Quellen, die in der Analyse erst zum Sprechen gebracht werden müssen, macht im Falle der vollständigen Vernichtung von Schrift den Nachweis meist sogar unmöglich, sofern er nicht durch Analogien oder durch die direkte Identifikation mit überlieferten Ereignissen also im Umweg über die „Schriftquellenhistorik“1 - erbracht werden kann. Im Falle der Siegel wird die Vergleichsbasis dadurch eingeschränkt, dass die traditionelle Sphragistik v. a. mit Archivalien arbeitet und daher auch in ihren hauptsächlichen Fragestellungen kaum Bezug auf archäologisch überlieferte Siegelstempel nimmt. ${ }^{2}$ Übergreifende wissenschaftliche Beobachtungen zur Materialität der Typare fehlen damit weitestgehend.

Gegenüber der regelhaft nachweisbaren Palimpsestierung mittelalterlicher Handschriften oder den zahlreichen historisch überlieferten Ereignissen von Bücherverbrennungen und -zerstörungen allgemein ${ }^{3}$ muss sich die folgende Darstellung auf einige verstreute Befundkontexte und eine bislang nicht adäquat publizierte, aber immerhin stetig wachsende Zahl materieller Relikte stützen. Zugleich wird sich jedoch zeigen, dass ein in den Schriftquellen nur selten auftretendes Phänomen sich

\footnotetext{
1 Der Begriff „Schriftquellenhistorik“ meint hier die akademische Geschichtswissenschaft in Gegenüberstellung zu anderen Teildisziplinen der Erforschung menschlicher Vergangenheit, die ihrerseits häufig als „,historische Hilfswissenschaften“ bezeichnet werden. Auch die Archäologie kann und sollte als historische Wissenschaft verstanden werden. S. hierzu Frommer 2007, $16 \mathrm{ff}$.

2 Andersen 2008, 71f. Bei den etwa 400 Typaren in dänischen Museumssammlungen handelt es sich zum Großteil um Bodenfunde, was laut Andersen auch auf die staatliche Gesetzgebung und die enge Zusammenarbeit mit privaten Sondengängern zurückzuführen ist.

3 Siehe hierzu näher im vorliegenden Band die Beiträge von Gereon Becht-Jördens, Georges Declerq und Marco Mostert.
}

Dieser Beitrag ist im Heidelberger Sonderforschungsbereich 933 „Materiale Textkulturen. Materialität und Präsenz des Geschriebenen in non-typographischen Gesellschaften“ entstanden (Teilprojekt A03 „Materialität und Präsenz magischer Zeichen zwischen Antike und Mittelalter“). Der SFB 933 wird durch die Deutsche Forschungsgemeinschaft finanziert. 
auf archäologischem Wege als mittelalterliches Brauchtum mit einer gewissen Verbreitung nachweisen lässt: Es geht um die Unbrauchbarmachung des persönlichen Siegels eines Verstorbenen durch Beschädigung oder Zerstörung.

Aus sprachlicher Sicht ist problematisch, dass sowohl das lateinische sigillum, ein Diminutiv von signum, als auch das hiervon abgeleitete deutsche Wort „Siegel“ nicht zwischen der negativen Urform und dem damit hergestellten positiven Abdruck unterscheiden. ${ }^{4}$ Auch die zumeist aus Wachs bestehenden Siegelabdrücke wurden im Mittelalter immer wieder absichtlich beschädigt, was meist mit einer Schmähung oder Missachtung des Textinhalts und damit auch des Ausstellers einherging. ${ }^{5}$ Im Folgenden soll aber ausschließlich die Zerstörung des Siegelstempels, auf Deutsch auch „Typar“ oder im Englischen „seal matrix“ genannt, betrachtet werden.

Bislang ist dieser Vorgang noch nicht unter dem Gesichtspunkt der Zerstörung von Geschriebenem betrachtet worden. Es muss daher zunächst kurz festgehalten werden, was das Siegel als Schriftträger auszeichnet und damit auch, wie sich diese Untersuchung in den Kreis der Arbeiten im vorliegenden Band einfügt: Geht man vom offensichtlichsten aus, so ist zunächst festzustellen, dass die meisten Siegelbilder eine Legende enthalten - oft in Form einer das Zentralbild umrahmenden Schriftzeile -, die meist der Be-Schreibung des Siegels selbst sowie seines Besitzers dient und dadurch die personelle Zuordnung ermöglicht. Damit hängt im Gegenzug die Bestimmung des Siegels als Authentifikationsinstrument anstelle der Person zusammen. Diese Funktion verweist darüber hinaus auf den Umstand, dass Siegel primär zur Beglaubigung von Urkunden eingesetzt werden und dadurch mit Schriftlichkeit in einem größeren Rahmen verbunden sind. Ihre Präsenz hat also zentralen Einfluss auf die Validität bestimmter Schriftstücke und ihre Beschädigung oder Vernichtung kann in letzter Konsequenz eine „Sinn-Zerstörung des Geschriebenen“ bedingen. ${ }^{6}$ Schließlich können auch die häufig anstelle von Personen oder Architekturen erscheinenden Wappendarstellungen in Siegelbildern ${ }^{7}$ als eine Form der Schrift verstanden werden: Im fortgeschrittenen Mittelalter hatte sich durch den Aufstieg der Ministerialen, die starke Zunahme von Familienwappen und persönlichen Wappen, deren häufige

4 Zur Terminologie s. Giele/Oschema/Panagiotopoulos 2015, 551ff.

5 S. hierzu Görich 2007. Ähnlich könnte auch der Fund einer intentional beschädigten Bleibulle des Gegenpapstes Paschalis III. (1164-1168) aus Trier interpretiert werden (Clemens 2005, 343ff.). Nicht gemeint ist hier das durch die Verschlussfunktion bedingte Erbrechen der nicht vor dem späten 12. Jh. belegbaren Brief- oder Sekretsiegel, das selbstverständlich nicht mit einer negativ konnotierten Symbolfunktion verknüpft werden sollte (Maué 2007, 183ff.). Dass das Konzept des Verschlusssiegels noch um 1000 eher ungebräuchlich war, legt z. B. die Interpretation des „Buches mit sieben Siegeln“ in der Bamberger Apokalypse (Staatsbibliothek Bamberg Msc. Bibl. 140, fol.1r) nahe: In der Miniatur sind die Siegel plan auf dem Vorderdeckel eines Kodex aufgebracht, wohingegen der biblische Text (Offb. 5 , 1) eine Schriftrolle meint, die siebenfach versiegelt gewesen sei.

6 Urkunden mit nicht mehr verwendeten Siegelbildern mussten daher häufig neu ausgestellt werden, damit sie ihre Rechtsgültigkeit behielten (s. Bedos-Rezak 2006, 339).

7 Zur Gestalt von Wappensiegeln zusammenfassend Ewald 1914, 205ff.; Pastoureau 1979, 231. 
Zusammenschiebung und das immer komplexere Repertoire von Symbolelementen auch die Heraldik zu einer eigenen Bilderschrift entwickelt, die von Spezialisten, den namensgebenden Herolden, gelesen und übersetzt werden konnte. ${ }^{8}$ Ein Siegelstempel kann also auf drei verschiedenen Ebenen mit der Sphäre des Geschriebenen in Beziehung stehen.

\section{Gestalt und Bedeutung mittelalterlicher Typare}

Sowohl die Siegelbilder als auch die Siegelstempel selbst durchliefen von der Spätantike bis zum Ausgang des Mittelalters eine umfassende formale Entwicklung. Zum Teil lässt sich diese in Bezug setzen mit geänderten Praktiken an und mit ihnen sowie mit einer Bedeutungsveränderung und zunehmenden Präsenz des Siegels als Machtinstrument. Verschiedene Siegeltypen sollen daher in chronologischer Folge kurz vorgestellt werden.

Aus dem frühen Mittelalter sind vereinzelt Siegelringe aus Edelmetall bekannt, ${ }^{9}$ die nach römischem Vorbild häufig ein Brustbild des Herrschers und in einer Umschrift Namen und Titel auf der Kopfplatte tragen. Der sicherlich bekannteste Ring diesen Typs wurde bereits 1653 im Grab König Childerichs († 481/82) im belgischen Tournai gefunden und zeigt das frontale Brustbild des Königs mit Lanze und Panzer (Abb.1.1). ${ }^{10}$ Die Umschrift CHILDERICI REGIS steht im Genitiv, bezieht sich also auf den Ring selbst als Insignie. Zeitgleich treten auch Siegelringe mit Monogrammen oder Namensringe mit seitenrichtiger Inschrift auf, so zum Beispiel zwei Stücke im Grab eines aus den Schriftquellen nicht bekannten ostgermanischen Fürsten im siebenbürgischen Apahida. Nur die Inschrift des Rings lässt vermuten, dass der Träger zumindest in latinisierter Form Omharus hieß. ${ }^{11}$ Die Ausstattung des Grabes ist ähnlich reich wie in Tournai und kunsthistorisch vergleichbar, was vermuten lässt, dass jener Omharus ein Zeitgenosse Childerichs und Verbündeter des römischen Imperiums war. Bei anderen Exemplaren solcher Goldringe, so auf dem Ring der Frankenkönigin Arnegunde aus St.-Denis bei Paris, sind nur einzelne Buchstaben seitenverkehrt eingraviert, sodass zwischen Siegel- und Namensring nicht immer sicher unterschieden werden kann. Hier ist zugleich auch ein Monogramm

\footnotetext{
8 Zur Entwicklung der Heraldik als Fachsprache im 13. Jh. s. Scheibelreiter 2006, 14f. u. 22f. sowie Pastoreau 1979, 198-202. Zu den Regeln der modernen deutschsprachigen Heraldik s. Biewer 2002.

9 Eine aktuelle Zusammenstellung frühmittelalterlicher Siegelringe (ohne Anspruch auf Vollständigkeit) bietet Weber 2014.

10 Wieczorek/Périn 2001, 172.

11 Wieczorek/Périn 2001, 56 und 156f. Zur Diskussion der Namenslesung und Parallelisierung mit Childerich vgl. Opreanu 2005.
} 
in der Mitte eingefügt, das je nach Interpretation entweder als Name aufgelöst wird oder als Genitiv REGIN(A)E. ${ }^{12}$

Mit der Karolinger Zeit endet die reiche Beigabenausstattung der Gräber im christlichen Mitteleuropa und damit ändert sich die Quellenlage völlig. Wir sind aber durch einzelne erhaltene Abdrücke an Urkunden darüber informiert, wie sich die Gestalt der Typare wandelt. Gerne werden antike Gemmen mit Kaiserporträts als Siegelbilder zweitverwendet oder Neuschöpfungen in antikisierendem Stil hergestellt. ${ }^{13}$ So ist auf zwei Siegeln Ludwigs des Deutschen (843-876) einmal ein antikes Porträt des Hadrian, im andern Fall aber der Karolinger selbst zu sehen. ${ }^{14}$ Der Abdruck einer Handhabe oder Öse im Wachswulst oberhalb des Bildfeldes lässt in manchen Fällen erkennen, dass diese Siegel nicht als Fingerringe getragen, sondern als separate Stempelplatten gestaltet wurden. Ein solches Gemmensiegel aus Bergkristall wurde in spätottonischer Zeit in Zweitfassung als Schmuckstein verwendet und ist so an einem Aachener Vortragekreuz überliefert (Abb.1.2). Aufgrund der Umschrift $+X P E$ ADIVVA HLOTARIVM REG kann es Lothar II. (855-869) zugeschrieben werden. ${ }^{15}$

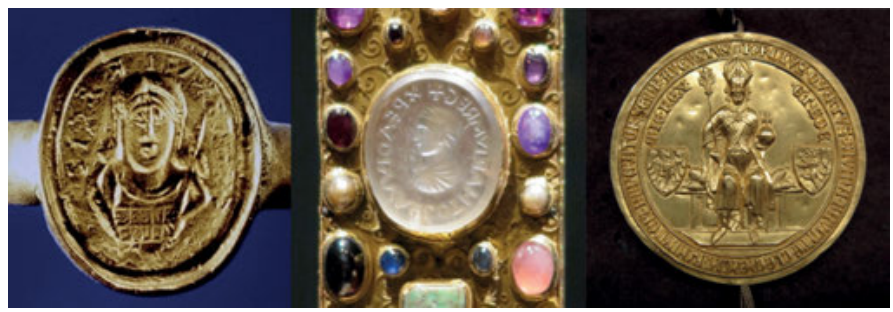

Abb.1: Königssiegel des Früh- und Hochmittelalters (von links nach rechts): 1. Ring des Childerich (Kopie in der Bibliothèque Nationale, Paris), 2. Gemmensiegel im Aachener „Lotharkreuz“, 3. Abdruck des Thronsiegels Karls IV. an der Trierer Goldenen Bulle von 1356 (Hauptstaatsarchiv Stuttgart). Zugleich sind hier die Überlieferungswege der Siegel dargestellt: Grab-/Bodenfund, Thesaurierung des Typars oder Archivierung des Abdrucks (@ gemeinfrei).

Bis in die Karolingerzeit war die Siegelführung stark restringiert und blieb nur den Königen und ihren Thronerben vorbehalten, ${ }^{16}$ erst etwa um die Mitte des 11. Jh. war der Gebrauch von Siegeln auch bei Bischöfen und Hochadeligen weit verbreitet. ${ }^{17}$ Mit der rapide wachsenden Zahl von Siegelführern in verschiedenen gesellschaftlichen Positionen prägten sich auch standesspezifische Bildtypen aus: So wurden ab der spätottonischen und salischen Zeit für Könige und Kaiser Thronbilder auf großforma-

12 Weber 2014, 111f.

13 Umfassend zu den Gemmen der Karolingerzeit s. Kornbluth 1995.

14 Ewald 1914, 184; Stieldorf 2004, 75.

15 Ewald 1914, 128.

16 Bedos-Rezak 2011, 84

17 Mit vorrangigem Bezug auf Frankreich: Bedos-Rezak 2011, 90. 
tigen runden Siegeln üblich (Abb. 1.3); ${ }^{18}$ im weiteren Verlauf des Hochmittelalters verbreiteten sich bei hochrangigen Adligen Siegel mit Reiterdarstellungen ${ }^{19}$ sowie v. a. im kirchlichen Bereich spitzovale Siegel, bei denen Bischöfe häufig auf der Kathedra sitzend, einfache Geistliche dagegen oft in betender Haltung mit ihren Schutzheiligen dargestellt werden. ${ }^{20}$ Verstärkt ab dem 13. Jh. rüsteten sich auch der Niederadel und schließlich das Bürgertum mit Siegeln aus, wobei im Bild (und auch in der Gesamtform) häufig die heraldischen Wappen nachgebildet bzw. im Falle der Bürger Hausmarken und allegorische Bilder gewählt wurden. ${ }^{21}$ Städte- und Institutionssiegel entwickelten sich ab dem Hochmittelalter ebenfalls in vielfältiger Form, blieben aber aufgrund ihres transpersonalen Bezugs oft jahrhundertelang unverändert in gültigem Gebrauch und stehen daher nicht im Fokus dieser Untersuchung.

Auch in der Gesamtgestalt der Siegelstempel zeigt sich eine Entwicklung hin $\mathrm{zu}$ komplexerer Formgebung (Abb. 2): Während Siegelringe in geringer Zahl kontinuierlich vorkommen, wird die Handhabe separater Typare zunächst nur durch eine Öse oder einen einfachen Steg gebildet. Erst in der Spätgotik kommen kurze verzierte Griffe $^{22}$ und schließlich im 15. Jh. Klappscharniere und figürliche Gestaltungen auf. Häufig werden die Siegel mit langen Gliederketten verbunden, die das Mitführen am Hals oder Gürtel ermöglichen. Das deutet sowohl auf ein zunehmendes und immer mobileres Urkundenwesen hin als auch darauf, dass die Siegel als Statussymbole gern zur Schau gestellt wurden. ${ }^{23}$ Lange Petschaftgriffe in der Art moderner Bürostempel werden dagegen erst in der frühen Neuzeit gebräuchlich - möglicherweise ein Hinweis darauf, dass die Siegel zunehmend in Schreibstuben und Kabinetten aufbewahrt und nicht mehr der Trageweise am Körper gemäß gestaltet wurden.

Die typologische Betrachtung lässt bereits erahnen, welch große Bedeutung Siegel im hohen und späten Mittelalter als Symbol der Amtsgewalt ihrer Träger erlangt hatten - ein Eindruck, der durch sigillographische Forschungen ${ }^{24}$ ebenso bestätigt wird wie durch Stimmen des Mittelalters selbst: So betont Konrad von Mure in der Summa de arte prosandi (1275/76), dass das Siegelbild dem Stand seines Trägers ange-

18 Ewald, 1914, 188ff.; Stieldorf 2004, 76f.

19 Die ersten bekannten Reitersiegel verwendeten Gottfried Martel von Anjou 1040/60 und Wilhelm der Eroberer 1069 (Stieldorf 2004, 79ff.). Ein typisches Beispiel ist auch das Siegel Rudolfs II. von Wittenberg (Abb.5).

20 Ewald 1914, 219; Stieldorf 2004, 72. Vgl. Abb.3.1.

21 Muhl 2003, 295ff.; Ansorge 2005, 95ff. Der früheste sichere Beleg eines heraldischen Wappens, nach den unklaren Schildzeichen auf dem Teppich von Bayeux (um 1070), ist das Reitersiegel des Gilbert „Strongbow“ de Clare, Earl of Pembroke, an Lansdowne Ms. 203 in der British Library London aus dem Jahr 1141 (Riquer 1983, 16).

22 Ansorge 2005, 95.

23 Pastoureau 1996, 285. Die persönliche Habe des Kaufmanns Hermann von Goch, die dieser am Tag seiner Hinrichtung 1398 trug, wird bis heute von der Stadt Köln aufbewahrt - darunter seine beiden an Gürtelketten hängenden silbernen Siegel (Diederich 2002, 109f. mit Abb.1; Muhl 2003, 299 Abb. 3). 24 Vgl. Adams/Cherry/Robinson 2008; Schofield 2015. 

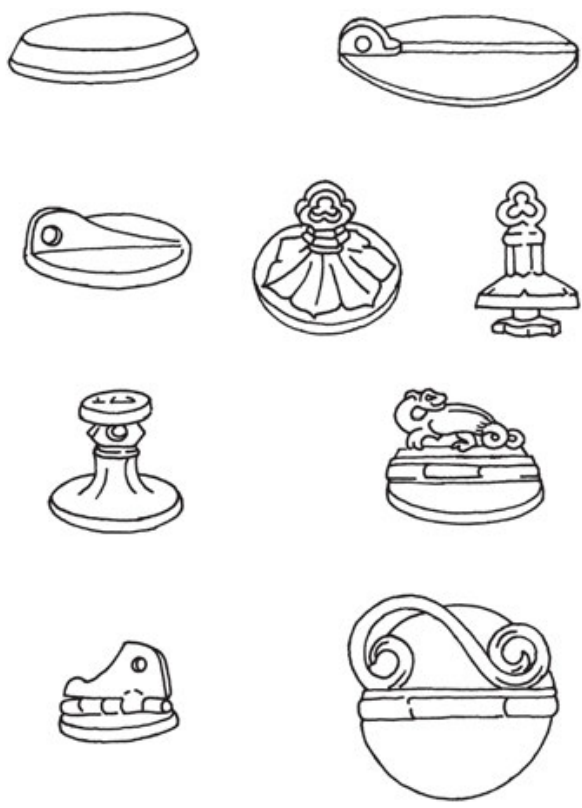

Abb. 2: Siegelformen und -handhaben bis zum Ausgang des Mittelalters (Umzeichnung des Verfassers nach Norberg 1970, 213).

messen sein müsse. ${ }^{25}$ Man könnte schließlich gar von den Siegeln als einer Personifizierung ihrer Inhaber sprechen. Brigitte Miriam Bedos-Rezak konstatiert in diesem Zusammenhang: „Seals were thus produced as avatars of their users [...]““.26

Diese enge Beziehung zwischen Siegel und Person wird auch darin deutlich, dass Finger- und Zahnabdrücke sowie Haarsträhnen anstelle des Siegels oder in Kombination mit diesem zur Beglaubigung von Urkunden eingesetzt werden konnten. ${ }^{27}$ Siegel formen demzufolge in Extension ihrer Inhaber - Bedos-Rezak spricht von ihnen gar als „Doppelgänger“28 - die mittelalterliche Wirklichkeit aktiv mit, sie konstruieren veritas. ${ }^{29}$

Konsequent weitergedacht, wirft diese Vorstellung eine Frage auf, die zum Abschluss der Überlegungen wieder aufgegriffen werden soll: Machte der Tod eines Siegelträgers damit nicht die Beseitigung seines Typars nötig, damit dieses ohne ihn nicht zum „Wiedergänger“ wurde? Zunächst soll aber auf den aktuellen historischen und archäologischen Forschungsstand zur intentionellen Beschädigung und Zerstörung von Siegelstempeln eingegangen werden.

25 Peltzer 2015, 63; Bedos-Rezak 2015, $94 \mathrm{f}$.

26 Bedos-Rezak 2011, 254. Den Körperbezug des Siegels stellt auch Achim Thomas Hack heraus, wenn er - in nicht ganz analoger Weise - die Theorie von den „zwei Körpern des Königs“ (Kantorowicz 1992) auf die beiden Bullenstempel des Papstes projiziert (Hack 2007, 53f.).

27 Pastoureau 1996, 284; Giele/Oschema/Panagiotopoulos 2015, 562f.

28 Bedos-Rezak 2006, 344.

29 Bedos-Rezak 2008, 1f. 


\section{Siegelzerstörung in der historischen Überlieferung}

Auch Schriftquellen zur Zerstörung von Siegeln im Funeralkontext ${ }^{30}$ wurden bislang nur selten systematisch untersucht - aus prozessualer Perspektive widmete sich diesem Thema bislang lediglich John Cherry; den mentalitätsgeschichtlichen Hintergrund hat v. a. Brigitte Miriam Bedos-Rezak herausgearbeitet. ${ }^{31}$ Beide Arbeiten konzentrieren sich wesentlich auf die früheste Überlieferung zu diesem Thema, nämlich das Prozedere um die Siegel des Bischofs von Durham, wie es zum ersten Mal 1095 anlässlich des Todes von William of Saint-Calais beschrieben wurde. Am detailliertesten ist die Schilderung der Ereignisse zum Jahr 1345: Nach dem Tod Bischof Richards von Bury werden seine vier silbernen Siegelstempel an den Altar des heiligen Cuthbert gestiftet und daraus ein silberner Messkelch gegossen. Dessen überlieferte Inschrift Hic ciphus insignis fit praesulis ex tetra signis Ri. Dunolmensis quarti, natu Byriensis weist explizit auf den Ursprung des Objekts als Seelstiftung hin. ${ }^{32}$ Auch wenn sich diese Stiftungspraxis zumindest bis zum Tod Thomas Hatfields 1381 erhielt, aus dessen Siegeln ein Votivbild angefertigt wurde, scheinen selbst in Durham einzelne Bischöfe hin und wieder Siegel ihrer Vorgänger weiterbenutzt zu haben. ${ }^{33}$

Auch aus der englischen Abtei St. Albans ist überliefert, dass die Siegel der Äbte am Tag der Beerdigung nach dem Hochamt öffentlich mit einem Hammer zerschlagen werden mussten. ${ }^{34}$ In der französischen Benediktinerabtei von Montivilliers wird zum Jahr 1255 ebenfalls vom Bruch des Siegels der verstorbenen Äbtissin berichtet. ${ }^{35}$ Besonders elaboriert erscheinen die Vorgänge um das von Maria Tudor geführte Großsiegel des englischen Königshauses, das nach ihrem Tod im Jahr 1558 zerstört wurde. Die Bruchstücke erhielt ihr Kanzler und Siegelbewahrer Nicholas Bacon, der daraus drei (!) Kelche für seine Söhne herstellen ließ - ein deutlicher Hinweis darauf, dass dieses Typar sehr massiv war. Alle Gefäße sind heute noch erhalten, zwei davon in Privatbesitz. Der sogenannte „Redgrave Cup“ im British Museum trägt am Rand der Cuppa eine umlaufende Inschrift: ${ }^{36}$

30 Vorgänge der Siegelzerstörung sind auch bei noch lebenden Inhabern überliefert, dienten dann aber, wenn es sich nicht um ein veraltetes und zu ersetzendes Typar handelte, ausschließlich der Degradation und/oder Entwürdigung (Cherry 2002, 83; Päffgen 2010, 219), wie im Fall Papst Johannes' XXIII. bei seiner Absetzung auf dem Konstanzer Konzil 1415 (Ewald 1914, 110; Hack 2007, 60).

31 Cherry 2002; Bedos-Rezak 2006.

32 Cherry 2002, 84.

33 Bedos-Rezak 2006, 337 u. $343 f$.

34 Cherry 2002, 85.

35 Dąbrowska 2011, 39.

36 Read/Tonnochy 1928, 102; Banister 1981, 278-79 fig. 2, zitiert nach http://www.britishmuseum. org/research/collection_online/collection_object_details.aspx?objectId=80389\&partId=1\&people=8 7656\&peoA=87656-1-4\&page=1 (Zugriff am 4.8.2016). 
A THYRDE BOWLE MADE OF THE GREAT SEALE OF ENGLANDE AND LEFT BY SYR NICHOLAS BACON KNYGHT LORDE KEEPER AS AN HEYRELOME TO HIS HOWSE OF REDGRAVE 1574

Über genaue Vorschriften bei solchen Prozessen sind wir nur durch die Kurie in Rom unterrichtet. Hier war zumindest im späten Mittelalter das Ritual der Siegelzerstörung minutiös festgelegt und betraf die massiven Prägestempel, mit denen der Papst die doppelseitigen Bleibullen ausstellen ließ. ${ }^{37}$ Im Zeremonienbuch des langjährigen päpstlichen Kämmerers François de Conzié (1383-1431) ist diesem Thema ein eigener Ordo gewidmet: Unmittelbar nach der Todesverkündung hat der Vizekanzler des Papstes von den Bullatoren die päpstlichen Siegelstempel zu konfiszieren und diese einzuschließen, nachdem er sie in Stoff gewickelt und wiederum mit seinem eigenen Siegel verschlossen hat. Er muss sie in einer eigens einberufenen Kardinalsversammlung vorzeigen, den Namensstempel mit einem Hammer zerschlagen und den Apostelstempel erneut wie zuvor verschließen. Das Bündel wird dann dem Kämmerer zur Obhut übergeben, der es nach der Wahl - noch versiegelt - dem neuen Amtsträger überreicht. ${ }^{38}$

Möglicherweise lässt sich die besondere Sorgfalt im Umgang mit den päpstlichen Typaren auch darin begründen, dass die mit ihnen gesiegelten Dokumente nicht nur weitreichende politische Konsequenzen haben konnten, sondern ihnen zum Teil auch ein maßgeblicher Einfluss auf das Seelenheil von Einzelpersonen zugesprochen wurde, wie sich unter anderem aus Grabfunden der bullae erschließen lässt. ${ }^{39}$

Urkundliche Vermerke der Siegelzerstörung anlässlich königlicher Begräbnisse sind in den Quellen nicht selten, bieten jedoch nur wenig detaillierte Beschreibungen ${ }^{40}$ - sie waren folglich für die Zeitgenossen wenig erklärungsbedürftig: Auch nach dem Tod Kaiser Sigismunds am 9. Dezember 1437 wird vom öffentlichen Zerschlagen der Siegelstempel berichtet, mit dem lapidaren Hinweis, dass es sich um die beim Tode ranghoher Fürsten übliche Sitte handle. ${ }^{41}$

In den von mir ausgewerteten erzählenden Quellen finden sich dagegen keine Hinweise für eine Beigabe der Siegelteile ins Grab. Die Spende oder Wiederverwertung zerschlagener Königssiegel scheint weitverbreitete Praxis gewesen zu sein. ${ }^{42}$ Gegenteilige archäologische Belege auf dieser sozialen Ebene sind nicht bekannt; ${ }^{43}$

37 Das Bildschema der päpstlichen Bullenstempel mit Namens- und Apostelseite (St. Paulus und Petrus) besteht seit Paschalis II. (1099-1118) bis heute unverändert (Hack 2007, 55).

38 Hack 2007, 56ff.

39 Clemens 2005, 352ff. Einige der Stücke waren wohl an (Ablass-)Urkunden anhängend beigegeben worden, in einigen Fällen (z. B. bei Durchlochungen) wurde evtl. auch dem Bleisiegel selbst eine Wirkung als Amulett oder Apotropaion zugeschrieben.

40 Cherry 2002, 83f.

41 Ewald 1914, $107 \mathrm{f}$.

42 Cherry 2002, 83f.; Bedos-Rezak 2006, 339 u. 348f.; Dąbrowska 2011, 39.

43 Cherry 2002, 86. 
die drei aus französischen Königsgräbern des 12. Jh. bekannten Typare wurden völlig unversehrt beigegeben. ${ }^{44}$

\section{Siegelzerstörung im archäologischen Befund}

Cherry publizierte im Zusammenhang mit seiner Schilderung der historischen Ereignisse auch bereits einzelne archäologische Funde aus Großbritannien, an denen sich die verschiedenen Formen der Siegelzerstörung - abgesehen von der vollständigen Desintegration - erkennen lassen: vom Zerschlagen des Typars über Abschabungen, Gravuren, Ritzungen oder Punzierungen der Stempelflächen bis hin zur gezielten vollständigen oder teilweisen Tilgung der Umschrift. ${ }^{45}$ Im Katalog der Siegelmatrices aus Frankreich im Münzkabinett der Bibliothèque Nationale in Paris stellte Ambre Vilain drei zerbrochene Siegel vor sowie das eines Klerikers Bernard, das mit sauber und regelhaft gesetzten Meißelschlägen entwertet wurde. ${ }^{46}$ Weitere physische Manipulationen, die an Siegelstempeln beobachtet werden können, sind das Verbiegen und Durchbohren. ${ }^{47}$ Während es sich bei den britischen archäologisch überlieferten Typaren mit Beschädigung stets um Einzelfunde ohne Kontext handelt und die französischen Stücke aus Altsammlungen ohne genaue Fundortangabe stammen, wurden Siegel oder Siegelbruchstücke vereinzelt auch direkt in Gräbern aufgefunden. In anderen Fällen stammen sie aus einem Friedhofsareal, woraus sich schließen lässt, dass sie ebenfalls im Rahmen des Bestattungsrituals deponiert wurden.

Diese Niederlegung im Grab ist an sich schon eine Besonderheit, da im christlichen Mitteleuropa etwa seit der Herrschaftszeit der Karolinger (751-911) die Ausstattung der Toten mit Tracht, Waffen, Schmuck, Möbeln, Geschirr, Speisen und weiteren Objekten unter dem Einfluss christlicher Begräbnisvorschriften und geänderter Jenseitsvorstellungen nahezu vollständig aufgegeben wurde. Im weiteren Verlauf des Hoch- und Spätmittelalters traten allerdings immer mehr „Ausnahmen“ von dieser Regel auf. Neben Zeugnissen christlicher Frömmigkeit und erhaltenen Trachtbestandteilen handelt es sich dabei v. a. um verschiedene Insignien der Macht in den

\footnotetext{
44 Meier 2002, 205f. Im Zusammenhang mit diesen Typaren Konstanzes von Kastilien († 1160), Ludwigs VII. († 1180) und Isabellas von Hennegau († 1190) wird auf ihre mögliche Funktion als Grabauthentiken hingewiesen (wofür Siegelbild oder Legende zumindest lesbar bleiben mussten).

45 Cherry 2002, 87f.

46 Vilain 2014, 57, 66, 87 u. 118. Bei dem Werk handelt es sich um die einzige bislang erschienene Publikation einer größeren Sammlung von Typaren in Frankreich.

47 Vilain (im Druck). Ich bedanke mich bei der Autorin für die freundliche Überlassung des noch unveröffentlichten Manuskripts.
} 
Gräbern kirchlicher und weltlicher Würdenträger, ${ }^{48}$ innerhalb derer die Siegel eine bislang kleine, regional sehr unterschiedlich auftretende Gruppe bilden. ${ }^{49}$

In Südskandinavien scheint die Beigabe von sowohl unversehrten wie auch beschädigten Siegeln besonders im späteren Mittelalter regelmäßig praktiziert worden zu sein $:^{50}$ Aus einigen Fundorten Dänemarks sind nämlich Typare bekannt, die in der Mehrzahl absichtlich beschädigt oder zerstört wurden. ${ }^{51}$ In den meisten Fällen sind hierbei Grabkontexte direkt nachgewiesen oder können durch die Nähe zu einem Bestattungsplatz wahrscheinlich gemacht werden. Allein im Chor der ehemaligen Franziskanerklosterkirche auf dem Axeltorv in Næstved (Seeland) wurden insgesamt 13 Siegel in oder bei Bestattungen aufgefunden, ${ }^{52}$ von denen elf Stücke entweder zerbrochen oder im Siegelbild durch Ritz- und Schlagspuren entstellt waren. Die beiden anderen Siegel waren so stark korrodiert, dass sich eine intentionale Beschädigung der Bildplatte weder feststellen noch ausschließen lässt. Soweit es die Lesbarkeit der Typare zulässt, können ihre Besitzer im lokalen Adel des späteren 13. und 14. Jahrhunderts vermutet werden. ${ }^{53}$ Nur das besterhaltene Stück, ein rundes Siegel mit Wappendarstellung und der Legende +S:NICHOLAI:HAC:MARSCALI, lässt sich eindeutig zuweisen: Es gehörte dem Marschall Niels Hak, der nach 1282 verstarb und dessen aus der heute südschwedischen (damals aber zum Königreich Dänemark gehörenden) Provinz Schonen stammende Familie zum dänischen Uradel gezählt werden kann. ${ }^{54}$

Darüber hinaus wurden in den letzten Jahrzehnten auch etwa 30 Siegelstempel von dänischen Burgen, also abseits des Grabkontextes, bekannt, von denen etwa ein Viertel absichtlich beschädigt wurde. ${ }^{55}$ Angesichts des Charakters der Burgen als Gerichtsorte führt Michael Andersen dies auf eine öffentlich deklarierte Unbrauchbarmachung nicht mehr genutzter Siegel zurück, während die vollständigen Stücke ihren Besitzern verloren gegangen sein könnten. ${ }^{56}$

48 Einen Überblick zur Grabausstattung weltlicher und geistlicher Eliten des fortgeschrittenen Mittelalters bieten Meier 2002 und Sanke 2012a und 2012b.

49 Eine Gesamtzahl europäischer Siegelfunde des Mittelalters aus Gräbern kann beim derzeitigen Forschungsstand nicht sinnvoll bestimmt werden. Eine Schätzung auf „knapp über zwanzig“ Stücke (Dąbrowska 2011, 31) ist jedoch sicher zu niedrig gegriffen.

50 Meier 2002, $207 \mathrm{ff}$.; Sanke 2012a, 443.

51 Liste der größtenteils unpublizierten Einzelfunde bei Meier 2002, 209f. Anm.120. Sieben der verzeichneten 18 Stücke (ohne den Fundort Næstved) wurden erkennbar intentional beschädigt. Zwei Neufunde aus Dänemark (einer davon ein Bruchstück) nennt Ansorge 2014, 147.

$52 \mathrm{Zu}$ den Ausgrabungen 1957 Skov 1959, $57 \mathrm{ff}$.

53 Meier 2002, 209 Anm.120. Auf den adligen Status der Bestatteten verweisen auch insgesamt vier spätmittelalterliche Schwerter aus Grabkontexten im Kircheninnenraum (Skov 1959, 67).

54 Skov 1959, 66.

55 Andersen 2008, 72.

56 Andersen 2008, 72f. 
Noch konzentrierter treten Siegelfunde bislang nur im ältesten Zisterzienserkloster Schwedens auf: Auf dem Gelände der 1143 gegründeten ${ }^{57}$ Abtei Alvastra (Provinz Östergötland) wurden bei zahlreichen Grabungskampagnen zwischen 1921 und 1955 insgesamt 25 Siegelstempel gefunden, dazu noch zwei Griffe und ein Halbfabrikat. Diese Typare wurden zuletzt 2005 von Elisabet Regner untersucht, die für die meisten Stücke einen ehemaligen Grabkontext ermitteln, sie jedoch nicht individuellen Bestattungen zuweisen konnte. ${ }^{58}$ Zum Teil mag dies mit der hohen Belegungsdichte und gegenseitigen Überschneidungen der Gräber in Friedhof und Kirche zusammenhängen; es könnte aber auch durch die nicht immer sicheren Kontextbeobachtungen bei den frühen Grabungskampagnen und die zerstreute Publikationslage ${ }^{59} \mathrm{zu}$ den jahrzehntelangen Forschungen in Alvastra bedingt sein. Mehr als die Hälfte aller geborgenen Exemplare wurde zerschlagen. Die drei ältesten Siegel aus Alvastra haben eine spitzovale Form (,Vesica“) und werden typologisch ins 13. Jh. datiert, wie das Typar eines Priesters Inge aus dem nahegelegenen Ort Heda, der möglicherweise mit einem urkundlich belegten Mann gleichen Namens aus dem Jahr 1294 identisch ist. Das Siegel zeigt eine Madonna mit Kind; nur die obere Hälfte wurde gefunden (Abb. 3.1).
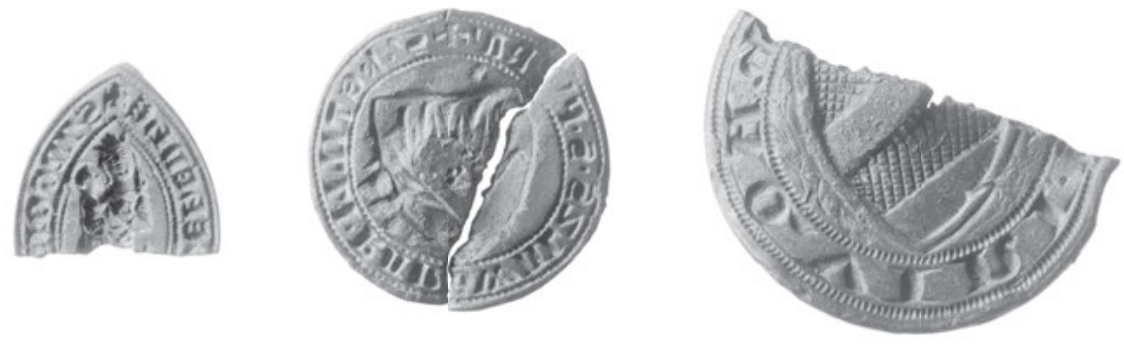

Abb. 3: Zerbrochene Siegel des 13. und 14. Jh. aus Alvastra (Schweden): 1. Inge aus Heda, 2. Ketill Ragvaldsson Puke, 3. Håkan Jonsson Lärna (C Riksantikvarieämbetet Stockholm).

Aus dem 14. und 15. Jh. wurden insgesamt acht Siegel gefunden, darunter die ebenfalls zerschlagenen Typare des Ketill Ragvaldsson Puke und des Håkan Jonsson Lärna (Abb. 3.2 und 3.3). Letzterer sicherte sich 1327 durch eine urkundlich belegte Stiftung seinen Begräbnisplatz im Kloster. Die restlichen Stücke stammen aus der beginnenden Neuzeit; mit der Reformation Schwedens im frühen 16. Jh. wurde das Kloster aufgelöst. ${ }^{60}$

57 Svartling 1969, 17.

58 Regner 2005, 193.

59 In Alvastra gefundene Pilgerzeichen und Bleibullen wurden bereits 1954 bzw. 1966 von Rune Norberg publiziert; zur Baugeschichte des Klosters s. Svartling 1969. Weiterführende Angaben bei Regner 2005, 194 u. $199 \mathrm{f}$.

60 Svartling 1969, 9. 
Insgesamt geben sich in den Siegeln Angehörige des niederen Adels und Klerus zu erkennen, deren Verbindung zu Alvastra zuvor nur teilweise aus den Schriftquellen bekannt war. Die konkrete Praxis der Siegelzerstörung in Alvastra und deren verschiedene Formen oder gar Motivationen werden von Regner leider nicht näher erörtert. Aus den zahlreichen Funden schließt sie aber, dass es sich dabei um eine nicht allzu ungewöhnliche Bestattungspraxis gehandelt haben muss. ${ }^{61}$

Ebenfalls in Südschweden wurde auch eine einzelne Bestattung aufgefunden, die dank gründlicher Untersuchung und günstiger Fundumstände den Vorgang am Grab besser nachvollziehbar macht. Im Dom zu Västerås (Provinz Västmanland) entdeckte man bei Ausgrabungen im südlich angebauten St.-Eriks-Chor in den 1950er Jahren die Familiengrablege des hochadeligen Sture-Geschlechts. ${ }^{62}$ Hier wurde der seit 1504 amtierende Reichsverweser von Schweden, Svante Nilsson Sture, im Jahr 1512 beigesetzt. Ins Grab beigegeben wurde ihm nicht nur ein Schwert von etwa 1,20 m Länge, sondern auch zwei seiner aus Silber gefertigten persönlichen Siegel, die jeweils zerschlagen wurden. Es handelt sich um ein großes Typar mit Kette und Löwengriff mit Scharnier, das einen Wappenschild mit Helmzier, Engeln und Namensumschrift zeigt, sowie ein kleineres Stück mit einfachem Wappenschild und Initialen, das vielleicht als Brief- oder Sekretsiegel diente. ${ }^{63}$ Auch wenn die einzelnen Bruchstücke durch das Absenken des Sarges oder das Einbringen späterer Bestattungen etwas bewegt worden sein könnten, lässt ihre verstreute Lage hier doch vermuten, dass sie in den geöffneten Sarg geworfen wurden (Abb.4).

Insgesamt sind von Svante Nilsson Sture fünf Siegelbilder an Dokumenten überliefert, die alle das heraldische Wappen in verschiedener Ausführung zeigen. Die beiden im Grab überlieferten Siegel sind zugleich die Typare, mit denen er in seinen letzten Lebensjahren urkundete. ${ }^{64}$ Man darf annehmen, dass er die früheren Ausfertigungen im Laufe seiner Amtszeit selbst ersetzen und vernichten ließ.

Aus dem deutschen Sprachraum liegen bislang keine systematischen bzw. überregionalen Arbeiten zum archäologischen Nachweis der Siegelzerstörung vor, obwohl bereits Wilhelm Ewald entsprechende Bodenfunde anführt - so aus St. Severin in Köln das Siegel des Kanonikers Andreas aus dem 13. Jh., das durch mehrere punktförmige Schlagmarken in der Bildfläche kassiert und seinem Besitzer vermutlich mit ins Grab gegeben wurde. ${ }^{65}$ Ähnlich verfuhr man offenbar mit den insgesamt sechs Siegeln des Magdeburger Erzbischofs Otto von Hessen (1327-1361), die allerdings bereits bei der Graböffnung von 1831 gefunden wurden und heute verschollen sind. ${ }^{66}$

61 Regner 2005, 200.

62 Nach Mannerstråle 1959, 2f., hatte Svante Nilsson über seine Mutter das Recht an der 1429 gestifteten St.-Eriks-Pfründe inne.

63 Mannerstråle 1959, 6f.

64 Andersson 1959, 35.

65 Ewald 1914, 109 u. Taf. 14 Abb. 3.

66 Lepsius 1842, 88f.; Ewald 1914, 109; Sanke 2012a, 441. Im Landesmuseum für Vorgeschichte Halle 


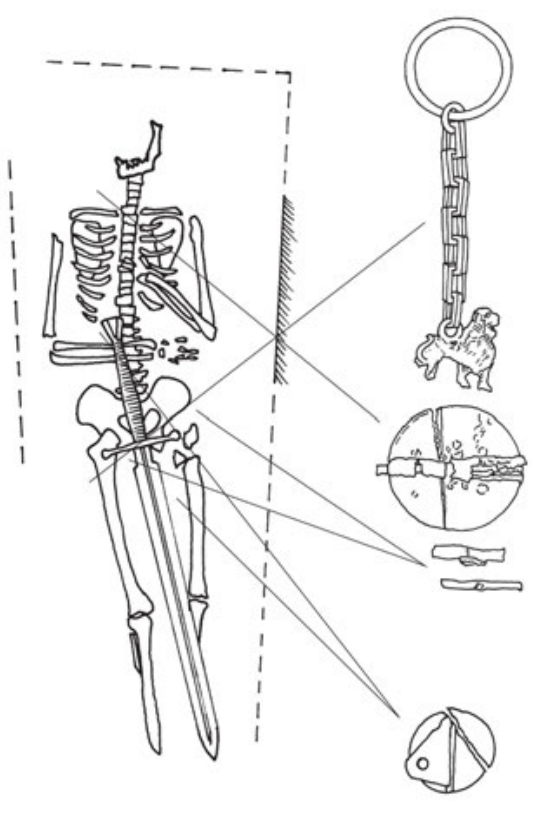

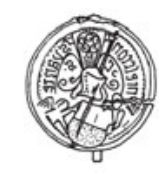

Abb. 4: Grab des Svante Nilsson Sture († 1512) in Västerås (Schweden) mit Lage der Siegelteile (Umzeichnung des Verfassers nach Mannerstråle 1959, 5 und Andersson 1959, 27 u. 28).

In einer Zusammenschau von Bodenfunden aus Mecklenburg-Vorpommern führte Jörg Ansorge auch ein Siegel mit durch Schlagmarken entstellter Bildfläche auf: Dieses um 1300 entstandene Typar des Ritters Albert von Bertekov wurde in der Verfüllung eines Kellers bei St. Marien in Pasewalk (Lkr. Vorpommern-Greifswald) gefunden, stammt also sehr wahrscheinlich nicht aus einem Grab. ${ }^{67}$

Vor wenigen Jahren erst wurde auf offenem Feld südlich von Stralsund das Fragment eines großen Siegelstempels gefunden, das dem Fürsten von Rügen Wizlaw III. (1302-1325) gehörte und erkennbar intentional zerbrochen wurde. ${ }^{68}$ Das Siegelbild ist an etlichen Urkunden aus den Jahren 1304 bis 1321 überliefert. ${ }^{69}$ Ein archäologischer

waren gegen Ende des 19. Jh. zumindest noch Gipsabgüsse der Siegel vorhanden. In der 2009 geborgenen Bestattung Ottos waren die Siegel nicht beigelegt (freundliche Mitteilung von Friederike Leibe, Halle).

67 Ansorge 2005, 96 mit Abb. 2. Nur ein Exemplar der Rostocker Franziskaner stammt vermutlich aus einem Grab, mehrere spätmittelalterliche Bürgersiegel dagegen aus Latrinenschächten (!). Insgesamt werden 14 mittelalterliche Siegel aus Mecklenburg-Vorpommern präsentiert, die mit angeführter Ausnahme unbeschädigt sind. Ein neuer Detektorfund aus Gützkow (Lkr. Vorpommern-Greifswald) liegt als (intentionales?) Bruchstück vor (Ansorge 2014, 147).

68 Ansorge 2014, 148.

69 Ansorge 2014, 149 mit Abb. 4-5. Der Autor übergeht dabei aber, dass Fürst Wizlaw III. mindestens ein weiteres zeit- und bildgleiches Typar besessen haben muss: Das gefundene Bruchstück ist zwar mit Stadtarchiv Stralsund Urk. 92 vom 9.6.1304 (Abb.4.1) identisch, weicht aber vom direkt daneben gezeigten Siegelbild an Stadtarchiv Stralsund Urk. 100 vom 12.3.1307 (Abb.4.2) in Details deutlich ab. Die Urkunde vom 10.5.1321 (Stadtarchiv Stralsund Urk. 169) stimmt mit dem Fundstück wieder überein (Ebd. 150, Abb.5). 
Kontext des Siegels ist dagegen nicht auszumachen und so muss offenbleiben, ob die Zerstörung anlässlich einer Neuanfertigung, des Todes oder erst zur Einsetzung seines Nachfolgers ${ }^{70}$ stattfand.

Neuere Funde zerschlagener Siegel sind auch aus Sachsen-Anhalt bekannt geworden: Das Bruchstück eines Klerikersiegels aus dem späteren 13. oder frühen 14. Jh. fand sich in einem 1510 angelegten Ossuarium am Merseburger Dom und muss demzufolge aus einem älteren Grab stammen. ${ }^{71}$ Eine personelle Zuordnung konnte hier nicht gelingen, da ein Großteil der Legende fehlt und erhaltene Abdrücke des Siegels bislang nicht bekannt sind. ${ }^{72}$

2009 schließlich wurde bei Ausgrabungen in der Franziskanerkirche in Wittenberg eine gemauerte Gruft mit der Bestattung Kurfürst Rudolfs II. (um 1307-1370), Herzog von Sachsen-Wittenberg, gefunden. ${ }^{73}$ Der Tote war mit seinem Schwert bestattet worden und man hatte ihm auch das durch Meißelspuren entstellte und in zwei Teile zerbrochene ${ }^{74}$ Großsiegel (Abb.5) auf die Brust gelegt. Diese Situation erinnert stark an das Sture-Grab in Västerås, jedoch steht eine detaillierte Publikation der Befunde noch aus.

Direkt aus einem Grab geborgen wurde auch das größere Bruchstück eines zerschlagenen Siegels Philipps II. von Hohenfels († 1290) in der Abteikirche von Otterberg (Lkr. Kaiserslautern). Den Schriftquellen zufolge unterhielt die Ministerialenfamilie von Bolanden-Hohenfels dort eine Familiengrablege. ${ }^{75}$

$\mathrm{Zu}$ erwähnen ist noch der bislang einzigartige Fund eines zerbrochenen Typars von einem jüdischen Friedhof auf dem Viehmarktplatz in Trier: Das vermutlich aus einem Grab stammende Siegel gehörte dem 1336 verstorbenen „Muskinus“ (Mosche bar Jechiel), der zeitweilig als Finanzverwalter des Trierer Erzbischofs tätig war, und zeigt an, dass sich die Zerstörung persönlicher Siegel nicht auf das christliche Milieu beschränkte. $^{76}$

Die unterschiedlichen Fundorte der Typare lassen den Schluss zu, dass nicht nur beim Tod des Inhabers allein Siegel zerstört wurden. Offenbar erforderte die Anschaffung eines neuen Siegels aus Verschleiß-, Mode- oder Standesgründen auch die ordnungsgemäße Entsorgung des Altstücks.

70 Ansorge 2014, 154.

71 Cottin 2008, 93.

72 Cottin 2008, 97.

73 Reichenberger 2009, „Grab von Kurfürst Rudolf II. entdeckt“, http://www.lda-lsa.de/fileadmin/ pdf/2009_02_26_rudolf-wittenberg.pdf (Zugriff am 27.7.2016); Gutjahr 2011.

74 Für die Bestätigung der intentionalen Zerstörungsspuren und Hinweise zur Materialität (das Siegel besteht aus Bronze, nicht wie ursprünglich angegeben aus Blei) danke ich Mirko Gutjahr, Wittenberg (mündl. Auskunft vom 28.6.2016).

75 Keddigkeit et al. 2015, 45f.

76 Clemens 2004, $174 \mathrm{f}$. 


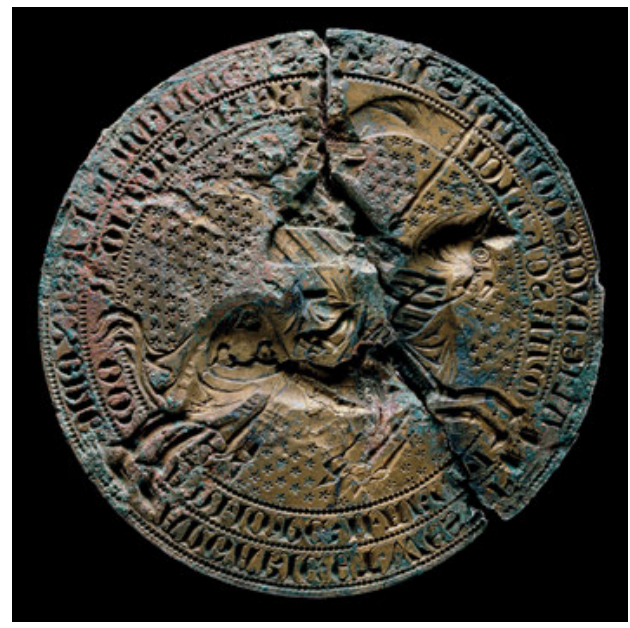

Abb.5: Großsiegel Rudolfs II. (1307-1370) aus der Franziskanerkirche in Wittenberg (mit freundlicher Genehmigung $\odot$ Landesamt für Denkmalpflege und Archäologie Sachsen-Anhalt, Juraj Lipták).

Auch die Tatsache, dass personenbezogene Typare in Archivkontexten deutlich seltener auftreten als Institutionssiegel, ${ }^{77}$ obwohl ihre ursprüngliche Zahl weit größer gewesen sein muss, spricht ex silentio für eine ehemals größere Verbreitung solcher Zerstörungsbräuche, als dies mit dem gegenwärtigen archäologischen und historischen Kenntnisstand nachvollzogen werden kann.

Dass der Wissensstand in naher Zukunft rapide anwachsen könnte, zeigt ein Blick in die Datenbank des „Portable Antiquities Scheme“78 in Großbritannien, eines staatlich organisierten, auf Eigeninitiative basierenden Programms, mit dem private Sammler und Schatzsucher ihre Fundstücke erfassen lassen können: Der Suchbegriff „seal matrix“ ergab in Kombination mit der gewählten Epoche „medieval“ am 5. Juli 2016 ganze 4374 Bodenfunde aus England und Wales. Details der Objekte werden aber nur unsystematisch aufgenommen - die kombinierte Freitextsuche für den Begriff „broken“ erzielte 474 Treffer, mit dem eindeutigeren Terminus „cancelled“ wurden dagegen nur 25 Typare bezeichnet. Grabkontexte wurden, wie bei Detektorfunden zu erwarten, in keinem Fall dokumentiert. Diese neu gewonnenen Bestände zu systematisieren, zu analysieren und die archäologischen Zusammenhänge zu klären, dürfte eine längerfristige Aufgabe sein. Die Erfassung kann allerdings bewirken, dass die Finder bereits selbst entsprechende Beobachtungen vornehmen und Fundplätze frühzeitig melden, um etwaige Kontexte zu sichern. Denn auch bei Altgrabungen ist leider gelegentlich zu erahnen, dass nicht die Befundsituation, sondern v. a. Umstände oder Qualität der archäologischen Untersuchung zu einem, bezogen auf die Kleinfunde, wenig aussagefähigen Gesamtbild geführt haben - wenn nicht lediglich die Publikation mangelhaft geblieben und Weiteres ohne Zugriff auf Originaldokumentation und Fundmaterial nicht nachzuvollziehen ist.

77 Cottin 2008, 97; Ansorge 2012, 150.

78 https://finds.org.uk/database/ (Zugriff am 5.7.2016). 


\section{Mögliche Motivationen eines Funeralbrauchs}

Nur in ganz seltenen Fällen, wie etwa beim Grab des Svante Nilsson Sture, lässt sich aus der dokumentierten Befundsituation unmittelbar auf das Geschehen bei der Bestattung schließen und sich dieses mit den historisch überlieferten Bräuchen der Siegelzerstörung parallelisieren. Auch in den Schriftquellen wird der Akt zwar gelegentlich beschrieben, die zugrundeliegende Intention jedoch nicht mitgeteilt, bis auf den Vermerk, Missbrauch und Urkundenfälschung verhindern zu wollen. ${ }^{79} \mathrm{Zum}$ Zweck der Liquidierung eines nicht mehr legal verwendbaren Authentifikationsinstruments wäre zwar ebenfalls eine öffentliche Inszenierung - wie sie anscheinend auch beim Typarwechsel zu Lebzeiten erfolgte ${ }^{80}$ - sinnig, jedoch erklärt sich die fallweise zu beobachtende Komplexität des Prozesses, wie sie besonders an den Beispielen aus Durham und Rom ${ }^{81}$ nachvollziehbar ist, hieraus ebenso wenig wie die Präsenz von Siegelbruchstücken in Gräbern. Daher soll abschließend der Versuch unternommen werden, weitere hypothetische Motivationen und Hintergründe der Siegelzerstörung aus Anlass des Todes zu diskutieren.

In der ur- und frühgeschichtlichen Archäologie ist die Präsenz rituell zerstörter oder unbrauchbar gemachter Objekte oder auch getöteter Tiere in und an Gräbern ein häufig wiederkehrendes kulturelles Motiv, ob es sich nun um verbogene eisenzeitliche Schwerter oder merowingische Pferdeopfer handelt. ${ }^{82}$ Dabei dürfte es sich um die Überreste von Trauerritualen handeln, die Arnold van Gennep im Rahmen seines Werks über die „rites de passage“ beschrieb. ${ }^{83}$ Christopher Daniell ging von einer grundsätzlichen Kontinuität solcher Zerstörungsbräuche im Funeralkontext durch das Mittelalter hindurch aus, auch wenn die Überreste der betroffenen Artefakte zumeist nicht mehr im Grab deponiert wurden. Für Daniell waren gerade Siegelstempel in herausragender Weise dazu geeignet, durch ihr Zerbrechen den Tod des Besitzers zu symbolisieren, ${ }^{84}$ da sie mit der Person des Trägers in engem Bezug standen. ${ }^{85}$

Die enge Verknüpfung eines Gegenstands mit seinem verstorbenen Besitzer, die wie im Falle der Siegel den Fremd- bzw. Weitergebrauch ausschloss, kann bis zur prin-

79 Ewald 1914, 107f; Dąbrowska 2011, 39.

80 Andersen 2008, 73.

81 Hack 2007, 56ff. Gerade im Falle des Papstsiegels konnte die Gefahr der Urkundenfälschung direkt mit Befürchtungen um das Seelenheil der Gläubigen (wie etwa bei Ausstellung ungültiger Dispense und Ablässe) verbunden sein.

82 Zahlreiche Beispiele bei Grinsell 1961, 476ff.

83 Gennep 2005, 158. Das Zerstören persönlicher Besitztümer des Toten wird dort unter die „Trennungsriten“ eingeordnet, jedoch lassen sich andere/spätere Opfer am Grab auch anderen symbolischen Dimensionen zuweisen.

84 Daniell 1997, $150 \mathrm{ff}$. Die Betonung der Siegel an dieser Stelle ist bemerkenswert, denn dem Autor war damals nur ein einziger Grabfund eines Siegelbruchstücks (aus St. Andrews in York) bekannt.

85 Bedos-Rezak 2011, 254; Giele/Oschema/Panagiotopoulos 2015, 558f. 
zipiellen Nichtvererbbarkeit des Objekts gehen. ${ }^{86} \mathrm{Um}$ es dem Diesseits und den Hinterbliebenen zu entziehen, konnte man es dem Toten beigeben und/oder zerstören.

Ebenso können beigegebene Siegel die spätere Identifizierung des Toten ermöglichen und damit als Grabauthentiken fungieren, sofern Bild und/oder Legende lesbar bleiben. ${ }^{87}$ Im Fall Niederadeliger mag die Motivation dahinter gewesen sein, durch diese Kennzeichnung des Leichnams das Begräbnisrecht an der von ihnen mit Seelstiftungen begünstigten Kirche auf Dauer zu sichern.

Schon allein aufgrund ihrer Funktion im mittelalterlichen Rechtswesen hatten Siegel auch eine hohe Bedeutung als Rangabzeichen oder Statusanzeiger. ${ }^{88}$ In diesem Zusammenhang ist bemerkenswert, dass bei der Bestattung des böhmischen Königs Ladislaus Postumus in Prag am 25. November 1457 nach der Totenmesse seine Siegel zusammen mit seinem Zepter, Reichsapfel und Schwert zerbrochen und seine Banner zerrissen wurden. ${ }^{89}$ Die Typare werden hier also in eine Reihe mit den anderen Zeichen königlicher Macht gestellt und ebenso behandelt ${ }^{90}$ - ausgenommen von solcher Zerstörung waren dagegen Insignien von transpersonaler Bedeutung, wie die von Karl IV. als Staatsjuwel des böhmischen Königsreichs in Auftrag gegebene Wenzelskrone. Möglicherweise diente das Zeremoniell hier dazu, die Sedisvakanz öffentlich zu demonstrieren, die im Fall von Ladislaus auch mit einem dynastischen Bruch einherging. ${ }^{91}$

Das Auftreten von Zerstörungsritualen anlässlich von Tod und Bestattung korreliert aber auch mit der generell zunehmenden Präsenz von Personifikationen des Todes im europäischen Spätmittelalter, die sich in einer Vielzahl von Totentanzdarstellungen und anderen Allegorien der Vergänglichkeit ausdrückt. Der Herrschaft des Todes hatten sich auch und gerade die Mächtigen zu unterwerfen. ${ }^{92}$ In diesem Sinne kann die Siegelzerstörung wie auch die Beschädigung oder gar Auslöschung des darin eingravierten Namens und Titels vielleicht auch als Demutsgestus verstanden

86 Härke 2003, 111f. Der Autor bezieht sich in seinen Beispielen zwar vorrangig auf die frühmittelalterlichen Angelsachsen, doch gerade für personenbezogene Siegelstempel ist eine solche Erklärung ebenso plausibel.

87 Meier 1997, 47; Meier 2002, 205ff.; Dąbrowska 2011, 38f.

88 Peltzer 2013, 237f.; Peltzer 2015; Stieldorf 2015, 212f. Zu Statusanzeigern im Begräbniskontext s. Härke 2003, 112f. Gerade im Falle der „Metamorphosen“ bischöflicher Siegel in Durham ist auch an eine „Gabe an [...] Gott“ als Motivation zu denken (ebd., 116).

89 Bláhová 1997, 106.

90 Hierin ist also keine rein „rechtliche Maßnahme“ zu sehen, wie dies Marie Bláhová vermutet (Bláhová 1997, 110).

91 Als frühneuzeitliche Parallelen für Zerstörungsrituale beim Erlöschen einer Familie im Mannesstamm lassen sich die Begräbnisfeiern des Hans Lasla von Kuenring am 9.4.1595 und des Peter Vok von Rosenberg am 1.2.1612 heranziehen: In beiden Fällen war jeweils ein schwarz gekleideter ,Trauerritter“ zugegen, der den Schild mit dem Familienwappen nach der Beisetzung zerschlug und in die Gruft warf (Bastl 1991, 264f. u. 266f.).

92 Kantorowicz 1992, 433ff.; Dinzelbacher 1997, bes. $50 \mathrm{ff}$. 
werden: Die Instrumente weltlicher Macht und irdischen Rechts würden also nicht nur gegenüber den Amtsnachfolgern, sondern auch in Erwartung der himmlischen Gerechtigkeit niedergelegt. $\mathrm{Zu}$ dieser geänderten Mentalität des Spätmittelalters passt der Befund, dass mit Absicht beschädigte Siegel in gesicherten mittelalterlichen Grabkontexten bislang nicht vor dem 13. Jh. aufgetreten sind. ${ }^{93}$

Die Zerstörung als Symbolakt hat Parallelen im mittelalterlichen Rechtswesen. Auch wenn das Stabbrechen durch den Richter als Bekräftigung des Todesurteils erst am Ausgang des Mittelalters belegt ist, ${ }^{94}$ findet sich doch, beispielsweise in der um 1300 entstandenen Heidelberger Handschrift des Sachsenspiegels, eine ähnliche Handlung im Kirchenrecht: In der Illustration zu Landrecht III Art. 63 § 2, in der die Auswirkung des Kirchenbanns erklärt wird, führt ein Priester die Exkommunikation durch, indem er eine Kerze über dem Delinquenten zerbricht. ${ }^{95}$ Das Erlöschen und Zerbrechen der Kerze bedeutet den Ausschluss aus der christlichen Heilsgemeinschaft.

Mit diesem Vergleichsbeispiel soll keinesfalls impliziert werden, dass das Zerbrechen des Siegels eines Toten dasselbe bedeuten würde oder in irgendeiner Weise mit Schmähung oder Entwürdigung zu konnotieren wäre. In beiden Fällen jedoch hängt der Symbolakt mit einem geänderten gesellschaftlichen Status der gemeinten Person zusammen, in unserem Fall durch den Tod des Siegelinhabers bedingt. Auch wenn die Lebenden und die Toten im Mittelalter durch Memoria und Liturgie miteinander eng verbunden waren und Tote durchaus als Rechtssubjekte angesehen wurden, ${ }^{96}$ mussten diese ihre irdischen Ämter und Güter dennoch an Rechtsnachfolger abtreten. Die aktive juristische Verfügungsgewalt bzw. die Aufgabe derselben könnte kaum besser symbolisiert werden als durch das Siegel und die Behandlung desselben.

Umgekehrt erhofften sich die Verfasser von Testamenten und Schenkungen vielleicht gerade durch die Zerstörung ihrer Siegel ein gesichertes Fortbestehen der von ihnen erlassenen Verfügungen, da diese nicht mit demselben Instrument abgeändert werden konnten. Gerade im Spätmittelalter, in dem sowohl die Siegelzerstörung als auch eine gesteigerte Sorge um die eigene Totenmemoria zunehmend greifbarer wird, ${ }^{97}$ mag diese Intention eine Rolle gespielt haben.

Aber auch unter dem Aspekt der Trauer und mit Bezug auf die Hinterbliebenen und die größere Gesellschaft kann der Symbolakt gedeutet werden. Auch heute geht es bei den Trauerritualen zwischen Ableben und Bestattung v. a. darum, den Tod

93 Vgl. die genannten Beispiele aus Südskandinavien und Deutschland. In England scheint sich nach archivalischen Quellen Ende des 13. Jh. dagegen ein starker Rückgang im Gebrauch persönlicher Siegel abzuzeichnen (Bedos-Rezak 2015, 95).

94 Ein früher Bildbeleg ist das um 1470 entstandene Fresko der Urteilsverkündung Christi in Thörl, Kärnten (Kocher 1992, 26).

95 Universitätsbibliothek Heidelberg, cod. pal. germ. 164, fol. 22v. Erläuterung der Szene bei MunzelEverling 2008, 94. In der 1336 entstandenen Oldenburger Handschrift sind die Kerzen deutlicher zu erkennen (Munzel-Everling 2013, 273, 279 u. 307 Abb. 26).

96 S. hierzu exemplarisch Oexle 1983.

97 Oexle 1983, 67f. 


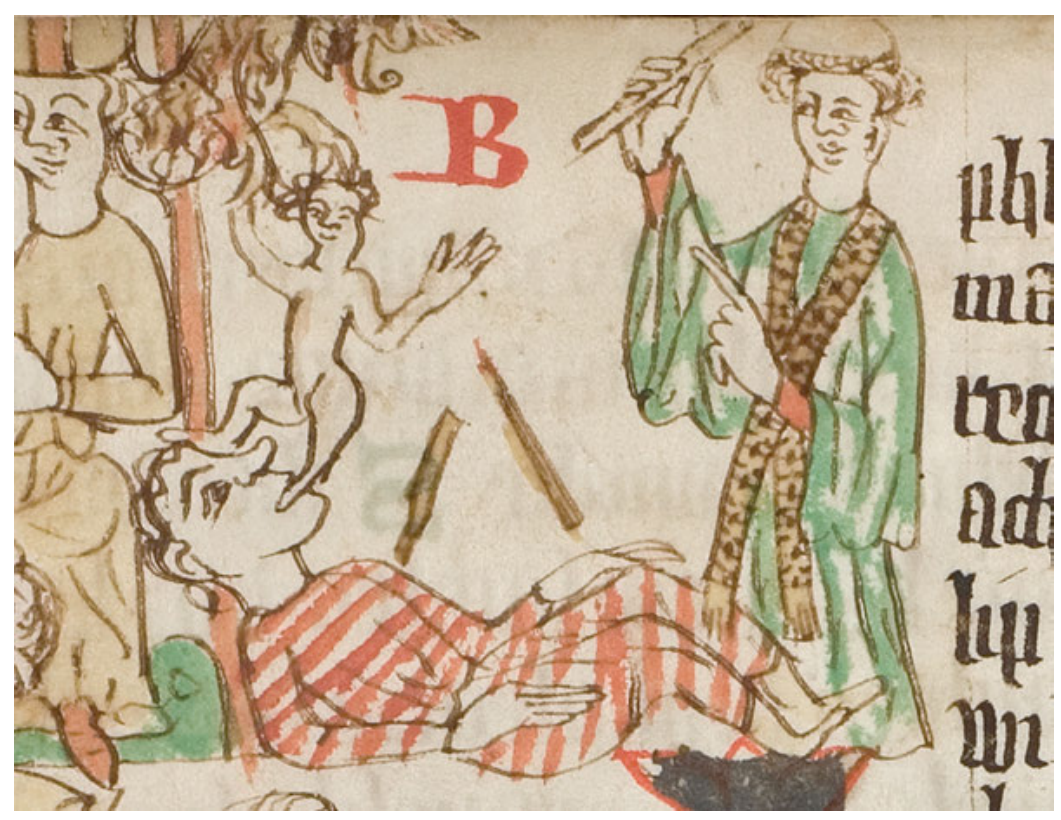

Abb. 6: Zerbrochene Kerzen im Kirchenbann. Heidelberger Handschrift des Sachsenspiegels, um 1300 (@ Universitätsbibliothek Heidelberg, Cod. Pal. Germ. 164, fol. 22v).

öffentlich ${ }^{98}$ und damit für alle erfahr- und begreifbar zu machen. Der bereits eingetretene physische Tod wird so um seine soziale Dimension ergänzt. Die verschiedenen Bräuche helfen - bezogen auf die individuelle Trauerarbeit - dabei, „den Weg zur ,letzten Reise` zu humanisieren“",99 dienen jedoch ebenso dazu, die durch den Tod bedrohte gesellschaftliche Ordnung aufrechtzuerhalten oder wiederherzustellen. ${ }^{100}$

Mehrere Motivationen eines Brauchs können parallel existieren, sich im Laufe der Zeit verändern oder in Vergessenheit geraten. Auch wenn sich diese kaum mehr im Detail erschließen lassen, haben die archäologischen Funde der letzten Jahrzehnte gezeigt, dass sich im Mittelalter die Beigabe von Siegeln in das Grab und deren Zerstörung nicht wechselseitig ausschlossen. Sie erscheinen daher keineswegs als so „ephemere und begrenzte Praktik[en]“, wie dies noch 2011 von Elżbieta Dąbrowska angenommen wurde. ${ }^{101}$ Zumindest für den west-, mittel- und nordeuropäischen Raum des späteren Mittelalters können wir also von einem Funeralbrauch mit einer

98 Guth 1989, 113.

99 Guth 1989, 118.

100 Ariès 1980, $775 f$.

101 Dąbrowska 2011, 40: „,...] l'inhumation des matrices de sceaux fut une pratique éphémère et limitée“. Eine Gesamtzahl intentional beschädigter Typare lässt sich beim gegenwärtigen Forschungsstand kaum bestimmen. Vilain (im Druck) erfasste bis 2013 etwa 300 Stück (ohne die Einträge im „Portable Antiquities Scheme“). 
gewissen Verbreitung ausgehen, auch wenn sich die bislang noch wenig erschlossene materielle Quellenfülle nicht im selben Maß in den Schriftquellen niederschlägt.

Die Zerstörung der Schrift im Siegel selbst scheint dabei in den meisten Fällen nur akzidentiell bedingt zu sein und mit der Zerstörung des Schriftträgers einherzugehen. Zugleich hatte der Siegelbruch aber auch Auswirkung auf Schrift in einem größeren Zusammenhang, nämlich die hiermit ausgestellten Dokumente, die nun nicht mehr im Namen des Toten modifiziert oder neu ausgestellt werden konnten. Damit war auch die Rechtsperson der Realperson in den Zustand der Totenruhe gefolgt. An die greifbare juristische Konsequenz dieses Funeralbrauchs schließen sich, wie zuvor skizziert, weitere Deutungen an, die in verschiedenste Richtungen führen und in vielen Fällen nebeneinander bestehen könnten. Auch wenn die genaue Interpretation des Geschehens letztlich offen bleiben muss, war die Wahl des Siegels als Ritualgegenstand sicher durch seine spezifische Aussagekraft zu Status und Person bedingt.

Dass eine derart starke ideelle Verbindung zwischen Träger und Insignie, wie sie sich an den mittelalterlichen Siegeln zeigt, auch gegenwärtig in entsprechenden Ritualen Ausdruck finden kann, zeigt sich besonders gut an einem ungewöhnlichen Fall: Seit 1265 ist der sogenannte Fischerring des Papstes belegt, ${ }^{102}$ der spätestens seit $1521^{103}$ nach dem Tod seines Trägers zerbrochen wird. Wegen des Amtsverzichts Benedikts XVI. zu Lebzeiten - einer historischen Ausnahmesituation ${ }^{104}$ - wurde dessen Ring aber nicht wie sonst üblich zerschlagen, sondern stattdessen nur die Bildplatte mit einer Gravur entstellt. ${ }^{105}$ Zwar trägt der Fischerring heute eine rechtsläufige Inschrift und lässt sich damit nicht mehr zum Siegeln verwenden - dennoch zeigt der besondere Umgang mit dieser Situation, dass Zeichen und Bezeichneter noch immer nicht so einfach voneinander zu trennen sind.

102 Waterton 1866, 138.

103 Waterton 1866, 40; Cherry 2002, 83.

104 Aus eigenem Wunsch als Papst abgedankt hatte zuletzt Coelestin V. im Jahr 1294.

105 http://kath.net/news/40416 (Zugriff am 4.8.2016). 


\section{Literaturverzeichnis}

Adams, Noël/Cherry, John / Robinson, James (Hgg.) (2008), Good Impressions. Image and Authority in Medieval Seals (British Museum Research Publications 168), London.

Andersen, Michael (2008), „Medieval Seal Matrices Found at Castles and Castle Mounds in Denmark: What Does Archaeology Tell Us about Their Use?“, in: Noël Adams, John Cherry u. James Robinson (Hgg.), Good Impressions. Image and Authority in Medieval Seals (British Museum Research Publications 168), London, 71-76.

Andersson, Aron (1959), „Svante Nilsson Stures sigill“, in: Fornvännen 54, 25-35.

Ansorge, Jörg (2005), „Siegelstempel und Siegel im archäologischen Fundgut“, in: Hauke Jöns (Hg.), Archäologie unter dem Straßenpflaster. 15 Jahre Stadtkernarchäologie in Mecklenburg-Vorpommern (Beiträge zur Ur- und Frühgeschichte Mecklenburg-Vorpommerns 39), Schwerin, 95-98.

Ansorge, Jörg (2012), „Mittelalterliche Siegelstempel aus dem Fürstentum Werle“, in: Archäologische Berichte aus Mecklenburg-Vorpommern 19, 150-156.

Ansorge, Jörg (2014), „Der Siegelstempel Wizlaws III. - letzter einheimischer Fürst von Rügen“, in: KulturERBE in Mecklenburg-Vorpommern 8, Schwerin, 147-154.

Ariès, Philippe (1980), Geschichte des Todes, München / Wien.

Bastl, Beatrix (1991), „Der gezähmte Tod. Bemerkungen zu den Riten um Sterben und Tod im österreichischen Adel der frühen Neuzeit“, in: Unsere Heimat. Zeitschrift des Vereins für Landeskunde in Niederösterreich 62, 259-269.

Bedos-Rezak, Brigitte Miriam (2006), „L'au-delà du soi. Métamorphoses sigillaires en Europe médiévale“, in: Cahiers de civilisation médiévale 49, 337-358.

Bedos-Rezak, Brigitte Miriam (2008), „In Search of a Semiotic Paradigm: the Matter of Sealing in Medieval Thought and Praxis (1050-1400)“, in: Noël Adams, John Cherry u. James Robinson (Hgg.), Good Impressions. Image and Authority in Medieval Seals (British Museum Research Publications 168), London, 1-7.

Bedos-Rezak, Brigitte Miriam (2011), When Ego Was Imago. Signs of Identity in the Middle Ages, Leiden/Boston.

Bedos-Rezak, Brigitte Miriam (2015), „Seals and Stars. Law, Magic and the Bureaucratic Process (Twelfth-Thirteenth Centuries)“, in: Phillipp Schofield (Hg.), Seals and Their Context in the Middle Ages, Oxford, 89-100.

Biewer, Ludwig (2002), Handbuch der Heraldik. Wappenfibel, Neustadt an der Aisch.

Bláhová, Marie (1997), „Die königlichen Begräbniszeremonien im spätmittelalterlichen Böhmen“, in: Lothar Kolmer (Hg.), Der Tod des Mächtigen. Kult und Kultur des Todes spätmittelalterlicher Herrscher, Paderborn / München, 89-111.

Cherry, John (2002), „The Breaking of Seals“, in: Michael Andersen u. Göran Tegnér (Hgg.), Middelalderlige seglstamper i Norden, Roskilde, 81-96.

Clemens, Lukas (2004), „Archäologische Beobachtungen zur Trierer Judengemeinde im Mittelalter“, in: Egon Wamers u. Fritz Backhaus (Hgg.), Synagogen, Mikwen, Siedlungen. Jüdisches Alltagsleben im Lichte neuer archäologischer Funde, Frankfurt am Main, 165-177.

Clemens, Lukas (2005), „Zeugen des Verlustes. Päpstliche Bullen im archäologischen Kontext“, in: Geschichtliche Landeskunde 59, 341-357.

Cottin, Markus (2008), „Ein mittelalterliches Siegelstempelfragment aus dem Kreuzhof des Merseburger Doms“, in: Harald Meller (Hg.), Merseburg - Seit 7000 Jahren ein guter Standort (Archäologie in Sachsen-Anhalt. Sonderband 8), Halle (Saale), 93-99.

Dąbrowska, Elżbieta (2011), „Les sceaux et les matrices de sceaux trouvés dans les tombes médiévales“, in: Marc Gil u. Jean-Luc Chassel (Hgg.), Pourquoi les sceaux? La sigillographie, nouvel enjeu de l'histoire de l'art, Villeneuve d'Ascq, 31-44.

Daniell, Christopher (1997), Death and Burial in Medieval England 1066-1550, New York. 
Diederich, Toni (2002), „Mittelalterliche Siegelstempel des Rheinlandes. Beobachtungen und Schlussfolgerungen“, in: Michael Andersen u. Göran Tegnér (Hgg.), Middelalderlige seglstamper i Norden, Roskilde, 107-128.

Dinzelbacher, Peter (1997), „Die Präsenz des Todes in der spätmittelalterlichen Mentalität“, in: Lothar Kolmer (Hg.), Der Tod des Mächtigen. Kult und Kultur des Todes spätmittelalterlicher Herrscher, Paderborn / München, 27-58.

Ewald, Wilhelm (1914), Siegelkunde, München/Berlin.

Frommer, Sören (2007), Historische Archäologie. Versuch einer methodologischen Grundlegung der Archäologie als Geschichtswissenschaft (Tübinger Forschungen zur historischen Archäologie 2), Büchenbach.

Gennep, Arnold van (2005), Übergangsriten, Frankfurt am Main.

Giele, Enno/Oschema, Klaus / Panagiotopoulos, Diamantis (2015), „Siegeln, Stempeln und Prägen“, in: Thomas Meier, Michael Ott u. Rebecca Sauer (Hgg.), Materiale Textkulturen. Konzepte Materialien - Praktiken (Materiale Textkulturen 1), Berlin / München / Boston, 551-565.

Görich, Knut (2007), „Missachtung und Zerstörung von Brief und Siegel“, in: Gabriela Signori (Hg.), Das Siegel. Gebrauch und Bedeutung, Darmstadt, 121-126.

Grinsell, Leslie Valentine (1961), „The Breaking of Objects as a Funerary Rite“, in: Folklore 72, 475-491.

Guth, Klaus (1989), „Sitte, Ritus, Brauch. Bräuche um Tod und Begräbnis“, in: Archiv für Liturgiewissenschaft 31, 100-118.

Gutjahr, Mirko (2011), „Ein Spiegel der Geschichte. Das Franziskanerkloster in Wittenberg“, in: Harald Meller u. Alfred Reichenberger (Hgg.), Kulturgeschichten aus Sachsen-Anhalt, Halle (Saale), 200-201.

Hack, Achim Thomas (2007), „Die zwei Körper des Papstes ... und die beiden Seiten seines Siegels“, in: Gabriela Signori (Hg.), Das Siegel. Gebrauch und Bedeutung, Darmstadt, 53-63.

Härke, Heinrich (2003), „Beigabensitte und Erinnerung: Überlegungen zu einem Aspekt des frühmittelalterlichen Bestattungsrituals“, in: Jörg Jarnut u. Matthias Wemhoff (Hgg.), Erinnerungskultur im Bestattungsritual (Mittelalter Studien des Instituts zur Interdisziplinären Erforschungdes Mittelalters und seines Nachwirkens 3), München, 107-125.

Kantorowicz, Ernst (1992), Die zwei Körper des Königs. Eine Studie zur politischen Theologie des Mittelalters, Stuttgart.

Keddigkeit, Jürgen / Werling, Michael / Schulz, Rüdiger / Lagemann, Charlotte (2015), „Otterberg, St. Maria“, in: Jürgen Keddigkeit, Matthias Untermann, Hans Ammerich, Pia Heberer u. Charlotte Lagemann (Hgg.), Pfälzisches Klosterlexikon. Handbuch der pfälzischen Klöster, Stifte und Kommenden, Bd. 3 (Beiträge zur pfälzischen Geschichte 26.3), Kaiserslautern, 524-587.

Kocher, Gernot (1992), Zeichen und Symbole des Rechts. Eine historische Ikonographie, München. Kornbluth, Genevra Alisoun (1995), Engraved Gems of the Carolingian Empire, University Park.

Lepsius, Carl Peter (1842), „Sphragistische Aphorismen“, in: Neue Mittheilungen aus dem Gebiet historisch-antiquarischer Forschungen 6, 84-115.

Mannerstråle, Carl-Filip (1959), „Sankt Eriks kor och Sturegraven i Västerås domkyrka“, in: Fornvännen 54, 1-10.

Maué, Hermann (2007), „Siegel zum Verschließen von Briefen“, in: Gabriela Signori (Hg.), Das Siegel. Gebrauch und Bedeutung, Darmstadt, 181-188.

Meier, Thomas (1997), „Inschrifttafeln aus mittelalterlichen Gräbern. Einige Thesen zu ihrer Aussagekraft“, in: Guy de Boe u. Frans Verhaeghe (Hgg.), Death and Burial in Medieval Europe, Bd. 2 (Papers of the ,Medieval Europe Brugge“ Conference 1997), Zellik, 43-53.

Meier, Thomas (2002), Die Archäologie des mittelalterlichen Königsgrabes im christlichen Mitteleuropa (Mittelalter- Forschungen 8), Stuttgart. 
Muhl, Arnold (2003), „Beeidigt durch das Schragenkreuz. Bemerkungen zu einem hochmittelalterlichen Siegelstempel“, in: Harald Meller (Hg.), Jahresschrift für mitteldeutsche Vorgeschichte 86, Halle (Saale), 295-314.

Munzel-Everling, Dietlinde (2008), „Die Verwendung von Rechtssymbolen in der Heidelberger Bilderhandschrift des Sachsenspiegels“, in: Gernot Kocher, Heiner Lück u. Clausdieter Schott (Hgg.), Signa Iuris, Bd. 2, Halle (Saale), 81-120.

Munzel-Everling, Dietlinde (2013), „Die Besonderheiten der Heidelberger Bilderhandschrift des Sachsenspiegels“, in: Gernot Kocher, Heiner Lück u. Clausdieter Schott (Hgg.), Signa luris, Bd. 11 (Tagung Tangermünde, 22.-25. Juni 2011), Halle (Saale), 267-281.

Norberg, Rune (1970), „Sigillstampar. Sverige“, in: Kulturhistorisk leksikon for nordisk middelalder 15, Kopenhagen, 211-214.

Oexle, Otto Gerhard (1983), „Die Gegenwart der Toten“, in: Herman Braet u. Werner Verbeke (Hgg.), Death in the Middle Ages (Mediaevalia Lovaniensia Serie 1. Studia 9), Leuven, 19-77.

Opreanu, Coriolan Horaţiu (2005), „Childeric şi Omahar. Doi regi barbari federaţi ai Imperiului Roman târziu“, in: Zeno Karl Pinter, Ioan Marian Ţiplic u. Maria Emilia Ţiplic (Hgg.), Relaţii interetnice în Transilvania. Secolele VI-XIII (Bibliotheca Septemcastrensis 12), Sibiu, 7-15.

Päffgen, Bernd (2010), Die Speyerer Bischofsgräber und ihre vergleichende Einordnung. Eine archäologische Studie zu Bischofsgräbern in Deutschland von den frühchristlichen Anfängen bis zum Ende des Ancien Régime (Studia archaeologiae medii aevi 1), Friedberg.

Pastoureau, Michel (1979), Traité d'Héraldique, Paris.

Pastoureau, Michel (1996), „Les sceaux et la fonction sociale des images“, in: Jérôme Baschet u. Jean-Claude Schmitt (Hgg.), L'image. Fonctions et usages des images dans l'Occident médiéval, Paris, 275-308.

Peltzer, Jörg (2013), Der Rang der Pfalzgrafen bei Rhein. Die Gestaltung der politisch-sozialen Ordnung des Reichs im 13. und 14. Jahrhundert (RANK. Politisch-soziale Ordnungen im mittelalterlichen Europa 2), Ostfildern.

Peltzer, Jörg (2015), „Making an Impression: Seals as Signifiers of Individual and Collective Rank in the Upper Aristocracy in England and the Empire in the Thirteenth and Fourteenth Centuries", in: Phillipp Schofield (Hg.), Seals and Their Context in the Middle Ages, Oxford/Philadelphia, 63-76.

Regner, Elisabet (2005), „Sigillstampar och gravar från Alvastra kloster“, in: Fornvännen 100, 193-200.

Riquer, Martí de (1983), Heràldica catalana. Des de l'any 1150 al 1550, Barcelona.

Sanke, Markus (2012a), Die Gräber geistlicher Eliten Europas von der Spätantike bis zur Neuzeit. Archäologische Studien zur materiellen Reflexion von Jenseitsvorstellungen und ihrem Wandel. Textband (Zeitschrift für Archäologie des Mittelalters. Beiheft 25.1), Bonn.

Sanke, Markus (2012b), Die Gräber geistlicher Eliten Europas von der Spätantike bis zur Neuzeit. Archäologische Studien zur materiellen Reflexion von Jenseitsvorstellungen und ihrem Wandel. Tafelband (Textband Zeitschrift für Archäologie des Mittelalters. Beiheft 25.2), Bonn.

Scheibelreiter, Georg (2006), Heraldik (Oldenbourg historische Hilfswissenschaften 1), Wien / München.

Schofield, Phillipp (Hg.) (2015), Seals and Their Context in the Middle Ages, Oxford.

Skov, Erik (1959), „Næstved gråbrødrekloster“, in: Nationalmuseets Arbejdsmark 1959, 57-68.

Stieldorf, Andrea (2004), Siegelkunde, Hannover.

Stieldorf, Andrea (2015), „Hochadeliges Selbstverständnis in bildlichen Darstellungen bis 1200.

Das Beispiel von Siegeln und Münzen“, in: Jörg Peltzer (Hg.), Rank and Order. The Formation of Aristocratic Elites in Western and Central Europe 500-1500 (RANK. Politisch-soziale Ordnungen im mittelalterlichen Europa 4), Ostfildern, 201-229.

Swartling, Ingrid (1969), Alvastra Abbey. The First Cistercian Settlement in Sweden, Stockholm. 
Vilain, Ambre (2014), Matrices de sceaux du Moyen Âge. Départment des Monnaies, Médailles et Antiques, Paris.

Vilain, Ambre (im Druck), „Le devenir post mortem des matrices médiévales“, in: Christophe Maneuvrier, Jean-Luc Chassel u. Clément Blanc Riehl (Hgg.), Apposer sa marque. Le sceau et son usage autour de l'espace anglo-normand (Colloqium Cerisy, 4-8 juin 2013), Paris.

Waterton, Edmund (1866), „XI.-On the Annulus Piscatoris, or Ring of the Fisherman“, in: Archaeologia 40 (1), 138-142.

Weber, Axel (2014), Der Childebert-Ring und andere frühmittelalterliche Siegelringe (Studien zu Spätantike und Frühmittelalter 7), Hamburg.

Wieczorek, Alfred / Périn, Patrick (Hgg.) (2001), Das Gold der Barbarenfürsten. Schätze aus Prunkgräbern des 5. Jahrhunderts n. Chr. zwischen Kaukasus und Gallien (Publikationen des ReissMuseums Mannheim 3), Stuttgart. 